\title{
Otimização do Processo de Roteamento e Atribui- ção de Comprimento de Onda em Redes Ópticas Translúcidas Utilizando Conceitos de Computação Inteligente
}

\author{
Lima, V.S. \\ Escola Politécnica de Pernambuco \\ Universidade de Pernambuco \\ 50.720-001 - Recife, Brasil \\ verusca.telecom@gmail.com
}

\author{
Pereira, H. A. \\ Grupo de Pesquisa em Radiometria, De- \\ partamento de Engenharia Elétrica, Cen- \\ tro de Engenharia Elétrica e Informática \\ Universidade Federal de Campina \\ Grande \\ 58.429-900 - Campina Grande, Brasil \\ helder.pereira@dee.ufcg.edu.br
}

Resumo O serviço de roteamento se faz necessário nas redes de comunicações devido à existência de várias rotas possiveis entre os nós. Isso implica na necessidade de tomada de decisão sobre qual dos caminhos deve ser utilizado para cada mensagem a ser encaminhada. Com o avanço das redes ópticas, algoritmos de roteamento baseados somente em parâmetros como, por exemplo, número de saltos entre origem e destino, não são a melhor alternativa para estabelecer caminhos ópticos. Diante desse cenário, pesquisadores vêm aplicando técnicas de inteligência computacional no processo de roteamento, para que seja possível considerar diversas características da rede como, por exemplo, ruídos, ocupação, de forma mais eficiente que os algoritmos utilizados atualmente e, assim, os niveis de qualidade de serviço desejados possam ser garantidos

\footnotetext{
Abstract The routing service is needed in networks communications due to the existence of several possible routes between nodes. This implies the need for decision making about which roads should be used for each message being forwarded. With advances in optical networks, routing algorithms based only on parameters, eg number of hops between origin and destination, are not the best way to establish optical paths. Given this scenario, researchers have been applying computational intelligence techniques in the process of routing, so you can consider various network characteristics, eg, noise, occupation, more efficient than the algorithms currently used, and thus levels of service quality desired can be secured.
} 


\section{Introdução}

Diante do cenário atual das telecomunicações, existe uma forte tendência para utilização das comunicações ópticas. A maior parte das informações de longa distância, seja tráfego de redes de computadores (internet), seja dos serviços de telefonia, passa pelos grandes enlaces de telecomunicações de alta capacidade usados para interligar países e continentes (backbones ópticos) [1].

As fibras ópticas, que são estruturas finas e flexíveis constituídas de vidro ou plástico, permitem propagar o sinal de informação utilizando ondas de luz por longas distâncias [2]. Atualmente, sistemas de comunicações ópticas compõem a tecnologia que podem prover a maior banda de transmissão entre os sistemas de transmissão conhecidos atualmente. Por esse e vários outros fatores, a comunidade científica investe muita atenção nesta área, pois os sistemas de comunicações ópticas ainda possuem um grande potencial para novas descobertas e desenvolvimento. Os principais desafios nessas redes consistem em garantir a qualidade do sinal durante o processo de transmissão e utilizar algoritmos de roteamento e atribuição de comprimentos de onda (RWA-Routing and Wavelength Assignment) de modo a minimizar o percentual de chamadas bloqueadas devido à falta de comprimentos de onda disponíveis ou degradação da relação sinal-ruído óptico [3].

\section{Redes Ópticas}

Graficamente uma rede óptica é representada por um grafo onde os nós representam os usuários que necessitam se comunicar e os enlaces representam as ligações entre estes nós [4]. A figura 1 mostra essa exemplificação.

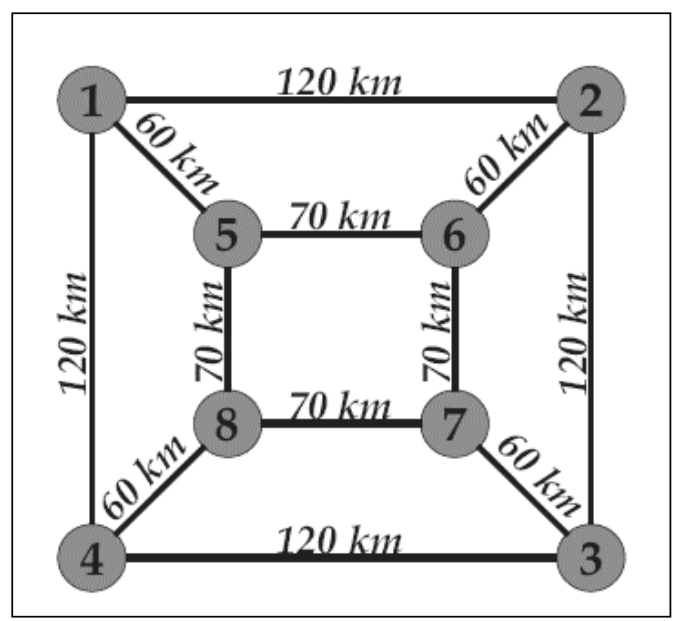

Fig. 1. Representação gráfica da topologia de uma rede óptica.
As redes ópticas têm como meio de transmissão as fibras ópticas. A fibra óptica por sua vez, corresponde ao meio onde a potência luminosa, injetada pelo emissor de luz (transmissor), é guiada e transmitida até o fotodetetor (receptor). Formada por um núcleo e por uma casca, ambos de material dielétrico, porém com índice de refração ligeiramente inferior ao do núcleo, a fibra permite a propagação da luz por reflexões sucessivas. Essa estrutura básica da fibra é envolvida por encapsulamentos plásticos de proteção mecânica e ambiental, formando o cabo óptico que pode conter uma ou mais fibras [2].

Quanto ao tipo, as redes ópticas podem ser definidas em três categorias: opacas, totalmente ópticas e translúcidas. As opacas são redes que utilizam a camada óptica apenas para transmissão. Dispõem de dispositivos eletrônicos que são capazes de realizar conversão eletro-óptica. Esses dispositivos são responsáveis por toda comutação e funções de gerenciamento. Uma das desvantagens desse tipo de rede é o alto custo para instalação, pois com o uso da tecnologia de multiplexação por divisão de comprimento de onda (WDM - Wavelength Division Multiplexing Optical Networks), que permite que vários comprimentos de onda, cada um carregando uma informação diferente, trafeguem numa mesma fibra simultaneamente, faz-se necessário para cada comprimento de onda um dispositivo conversor [4]. As totalmente ópticas ou ainda transparentes, são redes em que os dados são levados da origem até seu destino no domínio óptico, sem sofrer conversão eletro-óptica em nenhum nó da rede. Isso é possível graças aos dispositivos ópticos, que trabalham com os sinais no domínio óptico sem a necessidade de conversão do sinal. Essas redes apresentam restrições relacionadas às degradações do sinal óptico na camada física, uma vez que os dispositivos ópticos não são ideais e sem a presença de regeneradores ao longo do enlace é possível a degradação da comunicação devido ao acúmulo de penalidades geradas na camada física [4]. As translúcidas correspondem a um hibrido entre as opacas e as transparentes. São redes que apresentam dispositivos ópticos na sua camada física e apresentam regeneradores em alguns nós da rede [3].

\section{Algoritmos de roteamento e atribui- ção de comprimento de onda}

Em uma rede óptica, uma conexão é estabelecida através de um caminho óptico. Caminhos ópticos são conexões ópticas que vão de um nó origem a um nó destino utilizando um comprimento de onda nos enlaces que ligam esses nós [5].

A cada solicitação de conexão é necessário a escolha de uma rota e a alocação de um comprimento de onda na camada física. Esse processo de rotear e alocar comprimentos de onda é conhecido como problema de RWA. 
As redes ópticas WDM utilizam uma arquitetura baseada em caminhos ópticos para aproveitar a grande largura de faixa disponível para transmissão de dados. Nessa arquitetura o roteamento e a alocação de comprimentos de onda são efetuados procurando otimizar o uso dos recursos da rede, diminuindo o bloqueio das chamadas e o custo de implementação. O desempenho de uma rede WDM pode ser afetado por problemas no roteamento e alocação de comprimento de onda. Na tentativa de diminuir o impacto no desempenho da rede é preciso a existência de algoritmos de roteamento eficientes, garantindo assim uma baixa probabilidade de bloqueio. Técnicas baseadas em inteligência artificial e computação inteligente vêm sendo propostas na literatura. Essas técnicas visam desenvolver algoritmos de RWA eficientes [6].

\section{Roteamento por Série de Potências- PSR}

O roteamento por séries de potências (PSR - Power Series Routing) é uma ferramenta importante para o projeto de algoritmos de roteamento. Em [7] é proposta uma forma sistemática de se construir uma função de custo adaptativa para um enlace em uma rede. Baseia-se em um conjunto de parâmetros de rede determinados por um especialista. Ele está baseado na expansão de uma função de custo em uma série de potência.

Para a construção do PSR, inicialmente é feita a escolha das variáveis da função de custo do enlace. Um especialista em rede define quais parâmetros de rede devem ser levados em consideração na função de custo. Após a determinação desses parâmetros, a função de custo é expandida em uma série. Por fim, é feita a determinação dos coeficientes da série, esses coeficientes são encontrados pela técnica de otimização por enxame de partículas (PSO Particle Swarm Optimization) [7].

Em [7], a expansão da função de custo foi feita por uma série de funções multivariáveis que faz uso de um conjunto de polinômios ortogonais, da seguinte forma:

$$
f\left(x_{0}, x, \ldots, x_{k}\right)=\sum_{n 0=0}^{\infty} \sum_{n=0}^{\infty} \ldots \sum_{n k=0}^{\infty} b_{n 0, n 1 \ldots, n k} \bigsqcup_{j=0}^{k} x_{j}^{n j}
$$

Foram escolhidos como parâmetros de entrada, para a função de custo, a disponibilidade e o comprimento. Os valores foram normalizados para que todos os parâmetros estivessem na mesma escala, e assim as relevâncias dos parâmetros na função de custo fossem balanceadas de forma mais precisa. A razão de escolha desses parâmetros é que os mesmos têm alta correlação com o ruído acumulado ao longo do caminho óptico. Feita a escolha dos parâmetros de entrada, a função de custo é expandida a partir da seguinte equação:

$$
f\left(x_{i, j}, y_{i, j}\right)=\sum_{n 0=0}^{\infty} \sum_{n 1=0}^{\infty} b_{n 0, n 1} x_{i, j}^{n 0} y_{i, j}^{n 1}
$$

onde:

$$
x_{i, j}=\frac{\lambda_{i, j}^{a}}{\lambda_{i, j}^{T}}
$$

representa o parâmetro disponibilidade de enlace normalizado. O numerador e o denominador da equação (3) correspondem respectivamente ao número de comprimentos de onda não ativos e o número total de comprimentos de onda no enlace entre os nós $i$ e $j$, e

$$
y_{i, j}=\frac{d_{i, j}}{d_{\max }}
$$

representa o parâmetro comprimento de enlace normalizado. O numerador e o denominador da equação (4) correspondem respectivamente ao comprimento de enlace entre os nós $i$ e $j$ e o máximo comprimento de enlace na rede. A série é truncada para se obter uma aproximação com os $\mathrm{N}$ termos:

$$
f\left(x_{i, j}, y_{i, j}\right)=\sum_{n=00}^{N} \sum_{n=0}^{N} b_{n o, n 1} x_{i, j}^{n 0} y_{i, j}^{n 1}
$$

O PSR é finalizado com a utilização do PSO para obtenção dos coeficientes da série de potências que otimizam um parâmetro de desempenho de rede.

\section{Otimização por enxames de partícu- las}

O PSO foi proposto inicialmente por James Kennedy e Russel Eberhart durante uma pesquisa em que o objetivo inicial era simular o comportamento imprevisível de um bando de pássaros através de agentes inteligentes [8]. A técnica de otimização por enxame de partículas é inspirada na natureza e por isso é considerado por alguns como uma técnica de computação evolutiva. As técnicas de computação evolutiva são baseadas em mecanismos biológicos, que visam solucionar problemas de otimização combinatória - são problemas matemáticos complexos, onde a solução está associada a um alto custo computacional-, implementados em computador [9]. A busca por alimento e a interação entre os pássaros ao longo do vôo são modelados como um mecanismo de otimização [8]. Ao observar o comportamento de um bando de pássaros, verifica-se que o comportamento do grupo é influenciado pela experiência individual acumulada por cada indivíduo bem como pelo resultado da experiência acumulada pelo grupo. No PSO, cada candidato à solução do problema corresponde a um ponto no espaço de busca. O sistema é inicializado com um conjunto de soluções aleatórias, cada potencial solução é associada a uma partícula que possui uma velocidade, 
definida de forma aleatória, e se move em um espaço hiperdimensional em busca do melhor resultado possível [10]. As partículas movem-se no espaço de busca tendendo para uma combinação entre a melhor posição encontrada pela partícula (pbest) e a melhor posição encontrada pela vizinhança em que ela está inserida (gbest).

Uma partícula individual $i$ é composta por três vetores, são eles:

Sua posição no espaço D-dimensional:

$$
\vec{x}_{i}=\left(x_{i, 1}, x_{i, 2} \ldots x_{i, D}\right)
$$

A melhor posição encontrada por esta partícula:

$$
\vec{p}_{i}=\left(p_{i, 1}, p_{i, 2} \ldots p_{i, D}\right)
$$

E sua velocidade:

$$
\vec{v}_{i}=\left(v_{i, 1}, v_{i, 2} \ldots v_{i, D}\right)
$$

O deslocamento de cada partícula é representado pelo vetor resultante da composição de três forças representadas matematicamente em forma de vetor: inércia, memória e cooperação. O vetor inércia representa a força que impulsiona a partícula a seguir à direção a que tendia previamente. $\mathrm{O}$ vetor memória que representa o conhecimento da partícula, é responsável por fazer a partícula se direcionar aos lugares que se mostraram mais favoráveis ao problema em questão. $\mathrm{O}$ vetor cooperação representa a influência de um enxame em uma determinada partícula. Ele é responsável por direcionar a partícula para a melhor direção já conhecida pelo enxame [11]. As mudanças da posição das partículas durante a execução do processo são guiadas pela atualização da velocidade. As partículas voam pelo espaço de busca tendo suas velocidades atualizadas dinamicamente de acordo com o histórico das experiências individuais e coletiva de todo o enxame [12]. A cada iteração o algoritmo atualiza as posições e velocidades das partículas usando as seguintes equações:

$$
\vec{v}_{i, d}=v_{i, d}+c e_{1}\left(p_{i, d}-x_{i, d}\right)+c e_{2}\left(p_{g, d}-x_{i, d}\right)
$$

e

$$
x_{i, d}=x_{i, d}+v_{i, d}
$$

No algoritmo original, $c$ é uma constante com o valor dois, $p_{g, d}$ é a melhor posição encontrada pela melhor partícula no enxame e $e_{1}$ e $e_{2}$ são números aleatórios independentes com distribuição uniforme entre 0 e 1 gerados a cada iteração para cada partícula e cada dimensão de 1 à D [10].

Com o objetivo de melhorar o desempenho e a eficiência do algoritmo de PSO, pesquisadores propuseram alterações na forma como é feita a atualização de velocidade das partículas [13]. Introduzindo um fator de inércia $w$ (inertial factor) é possível obter um melhor balanço entre a busca global e local. A junção da equação da velocidade com o fator de inércia resulta na seguinte equação [13]:

$v_{i, d}=w v_{i, d}+c e_{1}\left(p_{i, d}-x_{i, d}\right)+c e_{2}\left(p_{g, d}-x_{i, d}\right)$

Outra melhoria proposta para ajudar na convergência foi a introdução do coeficiente de constrição (constricton factor) [14-15]. A adição do coeficiente de constrição $\chi$ à equação de atualização de velocidade das partículas foi proposta por Clerc e Kennedy [16]. O modelo de coeficiente de constrição propõe um novo método de escolha dos valores de $w, c_{1}$ e $c_{2}$ de forma que a convergência seja assegurada. Ele descreve que esses parâmetros passem a ser determinados analiticamente. A equação de atualização é apresentada da seguinte forma [14]:

$v_{i, d}=\chi\left(v_{i, d}+c e_{1}\left(p_{i, d}-x_{i, d}\right)+c e_{2}\left(p_{g, d}-x_{i, d}\right)\right)$

Em [7], focou-se no algoritmo PSO com o fator de constrição.

A figura 2 ilustra o pseudocódigo do algoritmo que foi utilizado para implementação do PSO [7].

O enxame é inicializado com valores de velocidade e posição gerados aleatoriamente, a função $g($ ) calcula a força (fitness) de cada partícula do enxame, avaliando o desempenho de cada uma, $\min _{i}\left(p_{\text {vizinho }}\right)$ retorna a posição $p_{\text {vizinho }}$ da partícula vizinha mais apta que tem o maior $g()$ dentre as duas possíveis. A quantidade de iterações que $o$ algoritmo deve executar foi adotada como critério de parada [7]. 


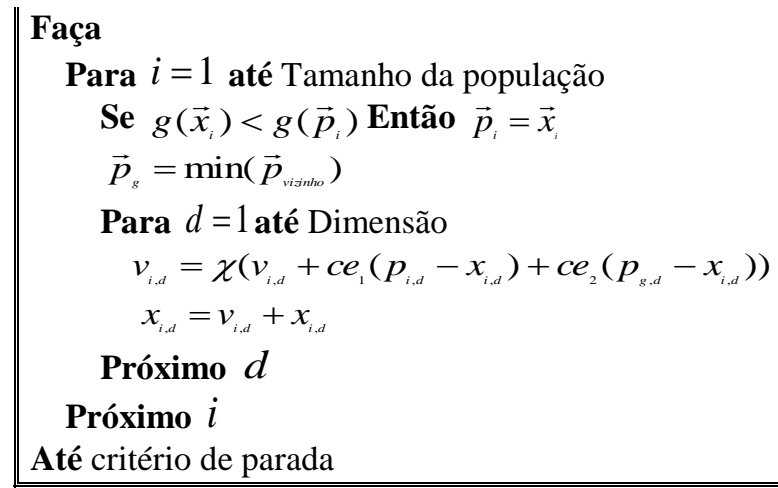

Fig.2. Pseudocódigo do algoritmo PSO utilizado.

\section{Proposta de Trabalho}

A utilização de redes de computadores para estudo, trabalho e negócios vem crescendo de forma exponencial nos últimos anos. As aplicações modernas precisam de uma transmissão rápida e eficiente, com menor perda possível de informação. Assim, serviços de rede devem ter qualidade para que as pessoas possam executar suas atividades de forma segura e adequada [17]. A tecnologia WDM é uma técnica promissora para atender a essa demanda crescente de largura de banda da rede. Com os avanços em tecnologia de comutação óptica as redes translúcidas surgem como candidatas promissoras para os futuros backbones da internet [18].

Uma rede óptica é opaca quando cada sinal óptico sofre regeneração eletrônica em cada nó intermediário na rede. A maior desvantagem desse tipo de rede é o alto custo. Entretanto, uma vantagem das redes opacas é que elas previnem o acúmulo de penalidades ao longo do caminho óptico. Já uma rede é dita totalmente óptica quando não existe regeneração eletrônica durante seu percurso fim-a-fim. Essas redes ainda suportam diferentes taxas de bits e de modulação. O principal inconveniente desse tipo de rede é que a mesma apresenta limitações de distância, devido às degradações na transmissão introduzidas por não linearidades, ruído acumulado, dispersão, etc. Com as redes ópticas translúcidas é possível alcançar uma performance próxima as das redes opacas, porém com diminuição nos custos. Elas representam um meio termo entre redes opacas e redes totalmente ópticas [3] [18].

O roteamento das chamadas é um dos fatores que mais afetam o projeto das redes ópticas e sua operação [3]. O problema de RWA é crucial para atender ao desempenho e qualidade dos novos serviços. Técnicas baseadas em computação inteligente vêm sendo propostas na literatura [7]. Essas técnicas visam desenvolver algoritmos de RWA eficientes.
Esse trabalho propõe a adaptação de um algoritmo de roteamento e alocação de canais (RWA) para redes transparentes utilizando uma técnica de computação inteligente e aplicando-o para o cenário de redes ópticas translúcidas.

\subsection{PSR-R uma solução inteligente para Re- des Ópticas Translúcidas}

O PSR foi proposto inicialmente e analisado em [7] para uma rede totalmente óptica. A idéia de utilizar o PSR em uma rede totalmente óptica é que o roteamento nesse tipo de rede deve levar em consideração as penalidades físicas. Uma vez não havendo regeneração ao longo dos caminhos ópticos o acúmulo dessas penalidades resulta em um grande prejuízo de transmissão. A utilização desses algoritmos de roteamento que levam em consideração o estado da camada física implica em maior complexidade computacional. A vantagem de utilizar o PSR é que o mesmo combina a simplicidade e a rapidez dos algoritmos de roteamento tradicionais com o alto desempenho conseguido pelos esquemas que levam em consideração as penalidades geradas na camada física [7].

O processo de construção do PSR inicia-se com a escolha dos parâmetros de entrada que devem ser levados em consideração na função custo. Para o PSR proposto para uma rede totalmente óptica em [7] foram escolhidos como variáveis de entrada os parâmetros disponibilidade e comprimento normalizado do enlace. O problema de roteamento em uma rede óptica translúcida também é referido como um problema de RWA [3]. Uma tarefa adicional do RWA utilizado nas redes translúcidas é que o mesmo também é responsável por utilizar regeneradores em alguns nós intermediários da rede. Diante desse cenário, o PSR-R surge como proposta para utilização no processo de roteamento de uma rede óptica translúcida. O PSR-R apresenta a mesma idéia do PSR utilizado em redes totalmente ópticas, a única diferença é que o PSR-R utiliza a mais, como variável de entrada, o parâmetro número de regeneradores no nó destino do enlace analisado, uma vez que a rede óptica translúcida é caracterizada pele presença de regeneradores em alguns nós estratégicos da rede.

\subsection{PSR-R uma solução inteligente para Re- des Ópticas Translúcidas}

As figuras 3, 4 e 5 apresentam respectivamente os pseudocódigos do simulador da rede translúcida, da função REGENERADOR_QoS e da função REGENERADOR_LAMBDA.

A arquitetura da rede translúcida aborda dois tipos de demandas para a regeneração. O primeiro tipo de exigência que surge é a qualidade do sinal. Essa é a razão mais comum para a regeneração [18]. Quando um caminho óptico percorre vários trechos de fibra óptica e componentes, 
a qualidade do seu sinal pode ser degradado. Cada componente em um nó intermediário pode apresentar perda de inserção. Os amplificadores de fibra óptica podem compensar alguma perda, mas ao mesmo tempo, introduzem ruído. Além disso, os comutadores geram interferência (crosstalk) entre os diferentes canais. Para superar essas deficiências, um caminho óptico pode recorrer à regeneração em um nó intermediário que apresenta essa função [18].

A contenção de comprimento de onda representa outro tipo de exigência para a regeneração. Ocorre quando a rota não disponibiliza um comprimento de onda fim-a-fim, ou seja, do nó fonte ao nó destino, e também quando dois caminhos ópticos, usando o mesmo comprimento de onda, são transmitidos na mesma fibra. Em uma rede WDM, a probabilidade de vários caminhos concorrerem para o mesmo comprimento de onda na mesma fibra pode ser bastante elevada causando uma quantidade significativa de bloqueio. Portanto, um regenerador torna-se uma alternativa viável para esse problema [18].

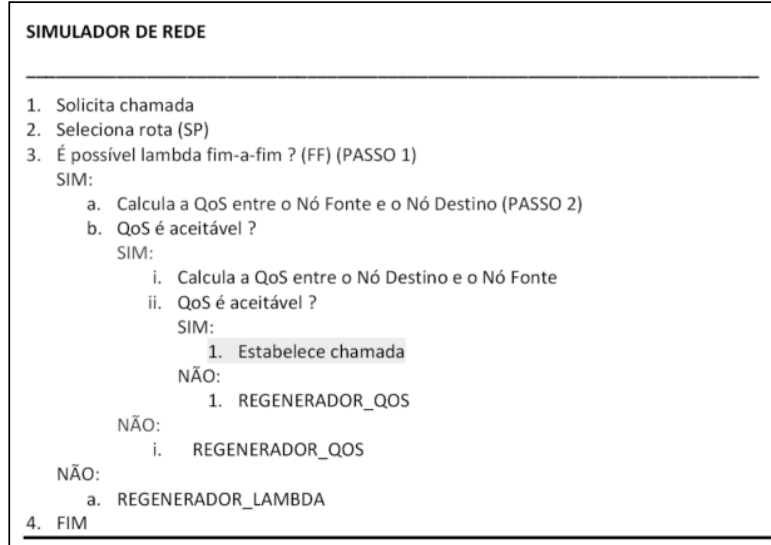

Fig. 3. Pseudocódigo do simulador de rede.

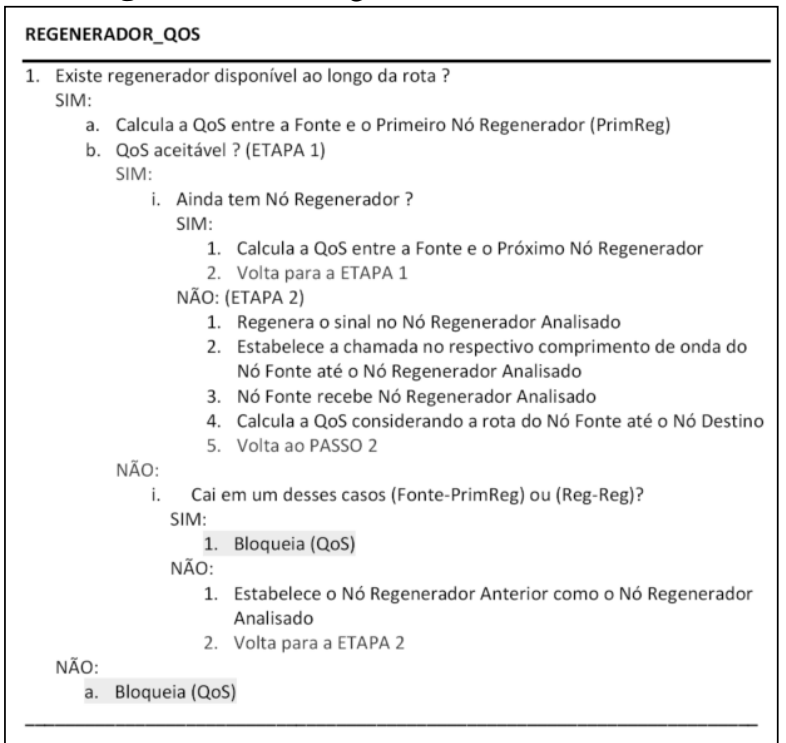

Fig.4. Função REGENERADOR_QoS.

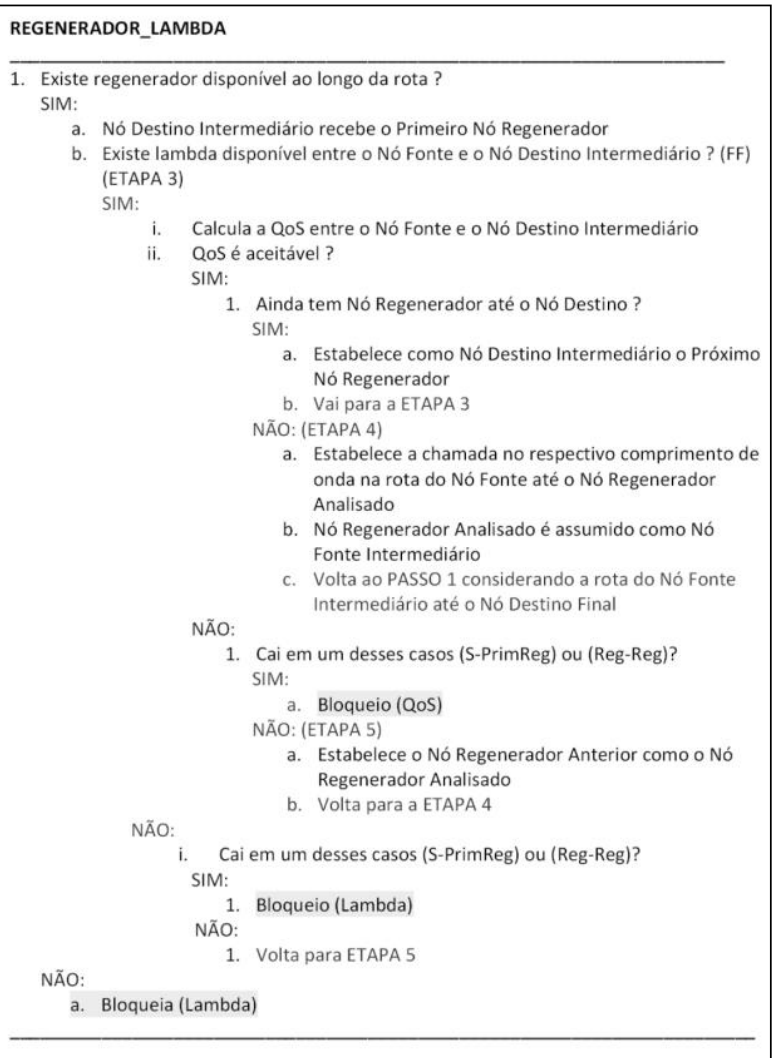

Fig.5. Função REGENERADOR_LAMBDA.

\section{Resultados}

O PSR-R foi proposto como uma solução para o roteamento em uma rede óptica translúcida. É aplicado para se construir a função custo adaptativa a ser utilizada nesse tipo de rede.

Com a escolha das variáveis de entrada da função custo, a mesma é expandida em uma série de potência. $\mathrm{O}$ PSR-R é descrito da seguinte forma:

$$
f\left(x_{i, j}, y_{i, j}, z_{i, j}\right)=\sum_{n=0}^{N} \sum_{n=0}^{N} \sum_{n 2=0}^{N} b_{n o, n 1} x_{i, j}^{n 0} y_{i, j}^{n 1} z_{i, j}^{n 2}
$$

onde:

$$
z_{i, j}=\frac{z_{i, j}}{z_{\max }}
$$


representa o parâmetro número de regeneradores. O numerador e o denominador da equação (14) correspondem respectivamente ao número de regeneradores no nó destino do enlace e o máximo número de regeneradores em um determinado nó da rede.

Finaliza-se o PSR-R com a determinação dos coeficientes da série utilizando o PSO, descrito na seção 5.

O procedimento de construção do PSR-R pode ser resumido de acordo com o esquema apresentado na figura 6 .

\begin{tabular}{|l|l|l|}
\hline ETAPA & DESCRIÇÃO & \multicolumn{1}{|c|}{ IMPLEMENTAÇÃO } \\
\hline Passo1 & $\begin{array}{l}\text { Escolha dos parâ- } \\
\text { metros de entrada } \\
\text { para a função de } \\
\text { roteamento }\end{array}$ & $\begin{array}{c}\text { Parâmetros escolhidos: ocupação ( } \\
\left.x_{i, j}\right), \text { comprimento }\left(y_{i, j}\right) \text { e número } \\
\text { de regeneradores }\left(z_{i, j}\right)\end{array}$ \\
\hline Passo2 & $\begin{array}{l}\text { Expansão da fun- } \\
\text { ção custo em uma } \\
\text { série }\end{array}$ & $\begin{array}{c}\text { Série de potências: } \\
f\left(x_{i, j}, y_{i, j}, z_{i, j}\right)=\sum_{n=0}^{N} \sum_{n=0}^{N} \sum_{n=0}^{N} b_{n o, n} x_{i, j}^{n 0} y_{i, j}^{n 1} z_{i, j}^{n 2}\end{array}$ \\
\hline Passo3 & $\begin{array}{l}\text { Determinação dos } \\
\text { coeficientes da sé- } \\
\text { rie }\end{array}$ & Uso do PSO \\
\hline
\end{tabular}

Fig.6. Construção do PSR-R.

\section{Conclusões}

O PSR-R foi implementado na plataforma computacional em [7], estima-se que sejam obtidos resultados satisfatórios para redes ópticas translúcidas em termos de probabilidade de bloqueio de chamadas.

\section{Referências}

[1] R. A. A. José, "Projeto de Sistemas de Comuni-

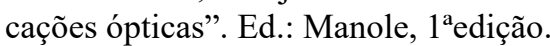

[2] C. A. Bastos-Filho, "Sistemas de Comunicações Ópticas. Disponível em: http://www.gta.ufrj.br/taquara/atividades/1.htm, acesso em 10 outubro de 2009.

[3] A. L. Souza-Filho, "Projeto de Redes Translúcidas de Longa Distância”. Dissertação (Mestrado em Engenharia Elétrica). Faculdade de Engenharia Elétrica e de Computação. Universidade Estadual de Campinas. Campinas: 30 de junho de 2003.

[4] D. A. R. Chaves, "Algoritmos Rápidos de IRWA para Redes Totalmente Ópticas”. Dissertação (Mestrado em Engenharia Elétrica). Universidade Federal de Pernambuco. Recife: 28 de fevereiro 2008.

[5] R. Ramaswami; K. N. Sivaraja, "Optical Networks (a practical perspective)". Califórnia:
Morgan Kauffmann Publishers Inc., 2002.

[6] H. Zang; J. P. Jue; B. Mukherjee, "A Review of Routing and Wavelength Assignment Approaches for Wavelength-Routes Optical WDM Networks". In.: Optical Networks Magazine. Vol. 1, $\mathrm{n}^{\circ} 1$, pp. 47-60. Springer Netherlands, janeiro 2000 .

[7] D. A. R. Chaves; D. O. Aguiar; C. J. A. BastosFilho; J. F. Martins-Filho, "Impairment Aware Routing Algorithm for All-Optical Networks Based on Power Series and Particle Swarm Optimization".

[8] Kennedy; R. Eberhart, "Particle Swarm Optimization". Inc Proc. Of the IEEE Int. Conf. on Neural Networks, pp. 1942-1948, Piscataway, NJ., 1995. IEEE Service Center.

[9] M. Poltosi, "Programando Soluções para Problemas de Otimização Combinatória". Congresso Simulado de Técnicas de Programação, São Leopoldo, 2006.

[10] D. Bratton; J. Kennedy, "Defining a Standard for Particle Swarm Optimization". Em: Swarm Intelligence Symposium, 2007 (SIS 2007) IEEE, pp. 120-127. Honolulu abril 2007.

[11] V. Miranda, "Computação Evolucionária Fenotípica". Porto, 2005.

[12] Y. Shi, "Particle Swarm Optimization". Em: Proceedings of the IEEE Neural Network Society. pp. 8-13, 2004.

[13] D. Corne; M. Dorigo; F. Glover, "New Ideas in Optimization", PP. 379-387. McGraw Hill, 1999.

[14] R. C. Eberhart; Y. Shi, "Comparing Inertia Weights and Constriction Factors in Particle Swarm Optimization". Em: Proceedings of the Congress on Evolutionary Computing, San Diego, USA, pp. 84-89, 2000.

[15] Y. Shi; R. C. Eberhart, "A Modified Particle Swarm Optimizer". Em: IEEE International Conference of Evolutionary Computation, Anchorage, Alaska, maio 1998.

[16] M. Clerc; J. Kennedy, "The Particle Swarm (explosion, stability and convergence in a multidimensional complex space)". Em: IEEE Transactions on Evolutionary Computation. Vol. 6, n 1 , pp. 58-73, fev. 2002.

[17] G. Torres, "Redes de Computadores Curso Completo". Ed. Axcel Books. Ed.: Axcel Books. Ano 2001.

[18] X. Yang, B. Ramamurthy, "Sparse Regeneration in Translucent Wavelength-Routed Optical Networks: 
Revista de Engenharia e Pesquisa Aplicada (2016) 2-1 : 85-92

Architecture, Network Design and Wavelength Routing”. p.p. 39-53, março 2005 